\title{
ANÁLISIS Y COMPORTAMIENTO ESPACIAL DE LA FRACTALIDAD TEMPORAL DE LA PRECIPITACIÓN EN LA ESPAÑA PENINSULAR Y BALEARES $(1997-2010)$
}

\author{
Óliver Meseguer-Ruiz' \\ Javier Martín-Vide ${ }^{2}$ \\ Jorge Olcina Cantos ${ }^{3}$ \\ Pablo Sarricolea ${ }^{4}$ \\ ${ }^{1}$ Dpto. de Ciencias Históricas y Geográficas, Universidad de Tarapacá. Grupo de Climatología. Universidad de Barcelona. \\ E-mail: omeseguer@uta.cl \\ ${ }^{2}$ Grupo de Climatología. Universidad de Barcelona. E-mail: jmartinvide@ub.edu \\ ${ }^{3}$ Instituto Interuniversitario de Geografía. Universidad de Alicante. E-mail: jorge.olcina@ua.es \\ ${ }^{4}$ Departamento de Geografía. Universidad de Chile. Grupo de Climatología. Universidad de Barcelona. \\ E-mail: psarricolea@uchilefau.cl
}

\section{RESUMEN}

La fractalidad temporal de la precipitación ' $\mathrm{D}$ ' indica la característica de autosimilitud de la lluvia, el hecho que los sucesos lluviosos se repitan con regularidad a diferentes escalas temporales. Los valores de D se determinan para 44 estaciones de la España peninsular y Baleares, variando entre 1,6039 y 1,4499. Según semestres, los valores de D son más bajos en la mitad cálida del año que en la mitad fría. En el norte los valores de D son mayores, a nivel anual y semestral, mientras que en la fachada oriental y Baleares y en el valle del Ebro son menores, y las mayores diferencias entre los valores de D delos periodos cálido y frío se dan en el norte y en el valle del Guadalquivir.

Palabras clave: Baleares, dimensión fractal, fractalidad temporal, Península Ibérica, precipitación.

Fecha de aceptación: octubre 2015. 


\section{ABSTRACT}

The temporal fractality of rainfall ' $\mathrm{D}$ ' indicates the property of self-similarity of rain, rainy events that recur regularly at different time scales. D values are determined for 44 stations of mainland Spain and the Balearic Islands, varying between 1.6039 and 1.4499. According semesters, D values are lower in the warm half of the year than in the cold half. In the north, D values are higher, annual and semi-annual, whereas in the Eastern coast and Baleares and in the Ebro valley are lower, and higher differences between D values of both warm and cold halves in the north and the Guadalquivir valley.

Keywords: Balearic Islands, fractal dimension, temporal fractality, Iberian Peninsula, precipitation.

\section{INTRODUCCIÓN}

La variabilidad del sistema climático en general, y de las variables atmosféricas en particular, es notable, a cualquier escala temporal que se considere. Esto es así para todas las variables climáticas, independientemente de la existencia o no de tendencias en su comportamiento cronológico. En la Península Ibérica, por su posición particular entre un océano y un mar casi interior, y en la franja fronteriza entre las zonas dominadas por los anticiclones subtropicales, al sur, y los vientos del oeste y las borrascas del frente polar, al norte, la variable que muestra una mayor dispersión en sus registros es la precipitación, tanto en las cantidades acumuladas como en el reparto temporal de la misma, lo que la hace ser un interesante objeto de estudio, como así lo confirma el gran número de trabajos que se han llevado a cabo (Casanueva et al., 2014; Goodess et al., 2002; Gonzalez-Hidalgo et al., 2009; de Luis et al., 2010; Martin-Vide et al., 2006; Rodríguez-Puebla et al., 1998; Rodríguez-Puebla et al., 2010; Sáenz et al., 2001 entre otros). El objetivo del presente trabajo es conocer en mayor medida el significado climático de la dimensión fractal aplicada a series de precipitación a resolución fina y poder, de este modo, diferenciar regiones dentro del área de estudio cuyo comportamiento de la precipitación a partir de este indicador sea homogéneo.

El mar Mediterráneo ocupa un área de aproximadamente 2,5 millones de kilómetros cuadrados entre Europa y África; y tiene únicamente una restringida comunicación con el océano Atlántico a través del estrecho de Gibraltar. A su vez, se subdivide en dos sub-cuencas, el Mediterráneo oriental y el Mediterráneo occidental, que se comunican a través del canal de Sicilia. Por su relativamente pequeña dimensión, su localización geográfica y por apenas tener salida al mar, el Mediterráneo es muy sensible y responde rápidamente a forzamientos atmosféricos y/o a influencias antropogénicas (Schroeder et al., 2012). Por otra parte, presenta comportamientos atmosféricos propios singulares, dada la protección y el aislamiento que le ofrece el relieve alrededor de su cuenca. El crecimiento demográfico, el turismo, el cambio climático y la sobreexplotación están ejerciendo una presión excepcional en el entorno mediterráneo y en sus ecosistemas y recursos. Además, se trata de una región donde los procesos oceánicos también tienen lugar, pero en unas dimensiones mucho meno- 
res que las que allí acontecen, como la formación de aguas profundas, que contribuyen a mantener una célula de circulación termohalina de dimensiones igual a las sub-cuencas, tal y como ocurre en el cinturón planetario a nivel oceánico (Schroeder et al., 2012). Esta realidad influye fuertemente en dotar a la región mediterránea de una marcada personalidad climática, como se verá a continuación.

Si se tiene en consideración la precipitación acumulada año a año, la evidencia sobre la variabilidad temporal es máxima. Tomando como referencia el observatorio de Gibraltar, en el extremo sur de la Península Ibérica, el de más antigüedad pluviométrica a nivel peninsular, que remonta sus datos a finales del siglo XVIII, es posible encontrar registros anuales próximos a los $2000 \mathrm{~mm}$ a mediados del XIX, frente a poco más de $350 \mathrm{~mm}$ a principios de la década de los 80 del siglo pasado. Es evidente la enorme variabilidad interanual del total de precipitación a lo largo de la serie, en la que el año más lluvioso totalizó más de cinco veces la precipitación del año más seco (Martín-Vide, 2008; Moreno et al., 1986, Wheeler et al., 1992). Otras muchas regiones en la Península Ibérica presentan una variabilidad similar a la que aquí se ha hecho mención, sobre todo en el sur y sureste peninsular, mientras que en los puntos más septentrionales, la variabilidad no presenta unos valores tan acusados. Pero esta dispersión no queda únicamente plasmada en un mismo punto a lo largo de los años, sino que a nivel espacial, y considerando la distribución estacional de la precipitación, es posible obtener una gran variedad de regímenes pluviométricos estacionales en España (Martín-Vide et al., 1998).

\section{I.1. Los fractales como base de estudio}

Fractal es una palabra inventada por Benoît Mandelbrot (1976) para reunir una clase de objetos que han desempeñado un papel histórico en el desarrollo de la Matemática del último tercio del siglo XX. Las formas y las estructuras geométricas regulares de Euclides y sus dimensiones naturales dan paso a formas nuevas, complejas, pero en las que subyace una regularidad ante los cambios de escala, con dimensiones fraccionarias. El concepto de fractal se utiliza para hacer referencia a objetos demasiado irregulares como para ser descritos según la geometría tradicional, pero que tienen la importante propiedad de ser invariantes por cambio de escala. La geometría fractal (Mandelbrot, 1976) es una extensión de la geometría clásica y engloba la descripción, la clasificación y el análisis de subespacios geométricamente "complicados". Generalmente, la estructura y organización de un conjunto fractal no hace posible especificar dónde -en lenguaje llano- se sitúa cada punto que lo compone. Por ello debe definirse alguna relación entre las diversas estructuras observadas en el mismo para varios niveles de resolución. Esta relación se formula cuantitativamente mediante el concepto de dimensión fractal, que describe el comportamiento ante los cambios de escala de las estructuras fractales. Si se toma un objeto con un tamaño lineal igual a 1 en una dimensión euclidiana $D$, y se reduce su tamaño por un factor de $1 / l$, se necesita un número $N=l^{D}$ de objetos autosimilares para cubrir el objeto original. Sin embargo, al despejar $D$, se obtiene:

$$
D=\frac{\ln N(l)}{\ln (l)}
$$


Existe una relación directa entre la dimensión fractal y el exponente de Hurst (Gneiding et al., 2004), y existen modelos estocásticos que afirman se expresa de la siguiente manera:

$$
D=n+1-H
$$

En este caso, $H$ es el exponente de Hurst, y $n$ es el número de dimensiones del espacio considerado (en este caso, 1, para series temporales). Cuando el exponente de Hurst es inferior a 0,5 , es decir, cuando la dimensión fractal es superior a 1,5 , el comportamiento de la serie se considera aleatorio.

$\mathrm{Al}$ igual que sucede con los objetos fractales, los procesos y sistemas invariantes por cambio de escala no poseen una escala que los caracterice. Teniendo esto en cuenta, un proceso fractal es aquel en el que el mismo proceso elemental tiene lugar a distintas escalas, es decir, en el que una parte reproduce el todo. Para el intervalo de escalas analizadas, los estadísticos del proceso siguen leyes potenciales caracterizadas por sus exponentes. La relación entre los estadísticos para las diferentes escalas consistirá simplemente en cocientes de escala. A modo de generalización, las dimensiones fractales, al contrario que las dimensiones corrientes o euclídeas, que son siempre números enteros no negativos ( 0 para el caso de un punto, 1 para una recta, 2 para un plano, 3 para un espacio en tres dimensiones, etc.), pueden presentar cualquier valor real no negativo. Para obtener una imagen más clara de esto, supongamos un punto que se desplaza sobre un plano describiendo un movimiento browniano, es decir, un movimiento aleatorio que tienda a completar o rellenar paulatinamente dicho plano. En un principio, su dimensión será 0, pero en el mismo instante en el que empiece a describir dicho movimiento, su dimensión fractal pasará a ser 1, e inmediatamente después, empezará a aumentar los decimales, pero sin llegar a dos, ya que no completará dicho plano.

Así pues, la geometría fractal y la dimensión fractal (como una dimensión no entera), es conocida como una herramienta valiosa que permite describir la forma de los objetos, y que ha ganado una amplia utilización y aceptación en muchos campos de las ciencias naturales incluyendo la Geografía, la Ecología o las nuevas tecnologías aplicadas a la información geográfica (Goodchild, 1980; Goodchild et al., 1987; Hastings et al., 1994; Peitgen et al., 1992). Los postulados de la geometría fractal han sido utilizados en la disciplina geográfica desde hace más de tres décadas. Fue introducido para medir la longitud de líneas costeras de las Islas Británicas (Mandelbrot, 1967), y, posteriormente, su uso se ha extendido de manera prolífica a multitud de estudios en topografía. Una serie de algoritmos matemáticos están ahora disponibles para determinar la dimensión fractal para entidades lineales y de área. Un examen detallado de la bibliografía muestra que no todas las características topográficas son fractales en todas las escalas estudiadas. Si bien la naturaleza de algunos fenómenos multifractales geográficos ha sido explorada en gran profundidad, no queda completamente entendido por qué algunos terrenos o superficies están mejor modelados con la geometría fractal que otros. Estos fenómenos multifractales hacen referencia a aquellos sistemas en los que un único exponente (la dimensión fractal) no es suficiente para describir su dinámica y es necesario un espectro continuo de exponentes.

El análisis fractal se ha utilizado con éxito para medir y caracterizar rasgos lineales irregulares como las costas, para describir y caracterizar las formas terrestres, y para regionalizar espacios estadísticamente según la forma de relieve. El análisis fractal también se puede 
utilizar para producir simulaciones de terreno con una dimensión conocida. Los resultados, en ocasiones poco precisos, derivan del hecho de que no existe una relación de uno a uno entre los procesos geomorfológicos y las formas de relieve que se generan (Gao et al., 1996). La metodología de análisis fractal también se ha aplicado desde hace algunas décadas a los estudios de carácter climático, y es que algunas de sus variables (temperatura, precipitación, presión atmosférica, entre otras) tienen un comportamiento fractal, tanto espacial como temporalmente, hasta el punto que determinan la persistencia de estas variables y sus respectivas dependencias mutuas (Rehman, 2009). A partir de las series de datos de las tres grandes variables climáticas (temperatura, precipitación y presión atmosférica) y de la variabilidad de la misma mes a mes y entre temporadas, es posible observar cómo los modelos climáticos regionales no son capaces de elaborar una buena predicción del clima local, ya que únicamente trabajan con cantidades promediadas. Así, aparecen hechos tan interesantes como que, en regiones como la India, las precipitaciones durante el monzón del suroeste se ven afectadas por la variabilidad de la temperatura y la presión del invierno anterior. En esta misma línea, otros índices de predicción que incorporan los fractales son más fiables ya que consideran más dinámicas climáticas, con lo que se deduce que estos nuevos modelos mejoran los existentes en una escala regional (Rangarajan et al., 1997; Rangarajan et al., 2004).

Sin embargo, en los últimos años han aparecido nuevos aportes que proponen distintos procesos de análisis para discriminar entre el comportamiento temporal de los datos generados por los modelos climáticos y las series climáticas reales obtenidas a partir de los registros de las redes de estaciones meteorológicas. Estos enfoques combinan el análisis de datos fractal y el seguimiento de los flujos de datos reales, y de aquellos generados por modelos, para detectar desviaciones en la correlación intrínseca entre las series de datos observados y los predichos por el modelo. Así, a partir de mediciones elaboradas por modelos climáticos regionales y las correspondientes mediciones de datos observados de una red de sensores analizada se muestra que este enfoque permite discriminar correctamente los datos, de lo que se desprende que todavía existe margen de mejora de los modelos de cambio climático, y que los conceptos basados en las teorías fractales pueden contribuir a ello (Nunes et al., 2011; Nunes et al., 2013).

En Paleoclimatología, el estudio de los sondeos o "cores" extraídos de los glaciares juega un papel fundamental a la hora de determinar cuál era el clima del planeta en el pasado (Pelletier, 1997; Valdez-Cepeda et al., 2003). El análisis de las burbujas de aire confinadas en el hielo revela cuál era la composición química de la atmósfera en el momento en el que esa burbuja quedó prisionera, así como permite conocer bajo qué condiciones se formó el hielo a partir de su nivel de compactación. Esto último se determina estudiando la conductividad del hielo a un impulso eléctrico conocido, y se ha descubierto que ésta es invariante de escala en tres órdenes diferentes de magnitud en la profundidad del núcleo de hielo. Este experimento llevado a cabo en la Antártida, a partir de un núcleo de hielo de 3.190 m de espesor, permite establecer conexiones climáticas entre los últimos 740.000 años, y, a partir del análisis fractal, suministrar información sobre la evolución de los ciclos glaciales (King, 2005). Estudios previos han relacionado la información obtenida a partir de núcleos de hielo de la Antártida con datos climáticos históricos obtenidos de los sedimentos marinos recogidos de las profundidades del mar (Raidl, 1996; Sahay et al., 1996). El análisis fractal de estos datos ya existentes aporta evidencias sobre las conexiones entre los datos climáticos de lugares geográficos muy distantes entre sí, como en Hungría (Bodri, 1994) o en la península de 
Kamchatka a partir de las erupciones volcánicas de origen explosivo de los últimos 10.000 años (Gusev et al., 2003), o, a menor escala temporal, a partir de registros de sedimentos en llanuras aluviales en el valle del Po (Italia) (Mazzarella et al., 2004).

En definitiva, es evidente que la mayoría de usos que se les ha dado a los fractales en los estudios de Climatología se han concentrado en metodologías de predicción en cuanto a modelos se refiere, y a la validación de los mismos, pero falta la aplicación de estos principios al comportamiento puramente dinámico del sistema climático, pero no por ello, inexistente, tal y como se analizará a continuación. Por la propia definición de un objeto fractal, es fácil inclinarse a pensar que la aplicación de los postulados de Mandelbrot ha discurrido por su aplicación a la distribución espacial de la precipitación siguiendo patrones que se ajustarían a objetos fractales. Incluso se ha planteado directamente la pregunta de si efectivamente es posible realizar una aproximación fractal a la misma (Sivakumar, 2001). El descubrimiento de estas nuevas realidades ha permitido avances en modelos de precipitación que han mejorado notablemente los existentes (Chou, 2003), llegando a simular campos de lluvia siguiendo la propiedad de multifractalidad, lo que certifica la invariancia por cambio de escala de este fenómeno. Ha quedado, pues, ampliamente demostrada las propiedades fractales de la distribución espacial de cantidades acumuladas de la precipitación. Para seguir avanzando en este conocimiento, es primordial conocer si la distribución temporal de la precipitación sigue estos mismos principios.

\section{I.2. La fractalidad temporal de la precipitación}

Los conceptos que se desprenden de la teoría fractal tienen una aplicación más intuitiva a la precipitación en su distribución espacial que en la temporal. Si bien cuando se hablaba de distribución espacial y fractales, de manera casi automática se puede pensar en que un campo de precipitación puede tener una forma fractal, y si se mira el detalle es posible comprobar que una parte representa el todo, respetándose la autosimilitud, o la invariancia por cambio de escala, cuando se habla de fractalidad temporal de la precipitación el concepto se hace más difícil de entender. En primer lugar, hay que partir de la base que el cambio de escala pasa en este momento a determinar si se ha acumulado lluvia en diferentes intervalos temporales de una duración determinada, y ver si este comportamiento se repite en intervalos temporales de mayor y menor duración. La lluvia, al tratarse de un proceso hidrológico no lineal, exhibe una alta variabilidad en un amplio rango de escalas temporales y espaciales. Las fuertes fluctuaciones temporales de la precipitación hacen que sea difícil su tratamiento estadístico y matemático.

Multitud de modelos se han desarrollado en Hidrología a partir de las propiedades fractales de la distribución temporal y espacial de la precipitación (Zhou, 2004; Khan et al., 2012). La utilidad de estos modelos de procesos hidrológicos de cuenca se incrementa considerablemente cuando pueden ser extrapolados a través de escalas espaciales y temporales. Este problema de la transferencia de escala, es decir, la descripción y la predicción de las características y los procesos a una escala diferente de aquella en la que se realizan las observaciones y mediciones, se ha convertido en el tema de mucha investigación actual en Hidrología y otras áreas afines. Efectivamente, este tipo de dinámicas se han identificado en estudios en la España peninsular a partir de series largas (noventa años) de precipitación acumulada anual, y su análisis revela que la distribución de esta variable se ajusta a una distribución fractal (Oñate Rubalcaba, 1997). Los valores obtenidos, con una media de 1,32 de dimensión 
fractal para todo el territorio, se encuentran en el mismo orden de magnitud que las dimensiones fractales obtenidas a partir de otros registros macrometeorológicos y paleoclimáticos.

El cálculo de la dimensión fractal a nivel anual también puede servir para identificar tendencias, que después han de ser confirmadas con algún otro tipo de técnica (como el test no paramétrico de Mann Kendall), con el fin de determinar si en el futuro, según los diferentes escenarios de cambio climático, las cantidades acumuladas serán mayores o menores que las actuales. Tal es el caso que se ha estudiado en la provincia de La Pampa (Argentina), donde se ha confirmado que las proyecciones realizadas por el IPCC-AR4 para esta región según los modelos se ajustan a la realidad de los datos observados (Pérez et al., 2009). Un estudio similar ha sido desarrollado en Venezuela (Amaro et al., 2004) a partir de datos de diez estaciones meteorológicas con valores de precipitación anual, y que se ajustan a una distribución fractal. Con estos resultados es posible predecir cambios climáticos a diferentes escalas temporales en Venezuela.

En otras regiones del globo, como la península de Shandong, en China, donde el problema del acceso a unos recursos hídricos cada vez más escasos es creciente al tratarse de una de las zonas de mayor y más rápido desarrollo del gigante asiático, el conocimiento de las tendencias de las precipitaciones se presenta como un tema crucial en el desarrollo futuro (Rehman et al., 2009; Gao et al., 2012). En la misma línea de los modelos multifractales, también se han llevado a cabo aplicaciones a estudios en la Península Ibérica, para el caso del observatorio de Córdoba, en el sur de España (Dunkerley, 2008; García-Marín et al., 2008). Sin embargo, se ha demostrado que las precipitaciones extremas se ajustan a modelos más complejos todavía que los multifractales, ya que se ven afectadas por períodos limitantes, como duraciones muy cortas o períodos de retorno muy largos (Langousis et al., 2009; Veneziano et al., 2002; Veneziano et al., 2006). En este tipo de estudios juega un papel determinante la resolución temporal con la que se trabaje, y es que el hecho de trabajar con datos horarios, por una parte, y con datos diarios, por otra parte, ya provoca cambios en los valores de las dimensiones fractales, también en parte por la influencia de la precipitación más característica de cada lugar (García, 2007; López, 2012). Además, este método de análisis de la fractalidad permite la discriminación de mejores métodos de análisis para las frecuencias de la precipitación, coincidiendo con estudios mencionados anteriormente (Gao et al., 2012), llegando incluso a poder definir el régimen de precipitaciones de una región concreta (Dunkerley et al., 2010; Kutiel et al., 2014, Reiser et al., 2010).

En la mayoría de los estudios sobre propiedades de escala en el proceso de precipitación, el comportamiento multifractal se ha investigado sin tener en cuenta los diferentes mecanismos de generación de lluvia involucrados. Sin embargo, se sabe que los procesos de lluvia están relacionados en ciertas escalas, determinadas por las características climatológicas, así como por características meteorológicas regionales y locales. Una de las implicaciones derivadas de estas correspondencias es la posibilidad de que los parámetros multifractales de la precipitación puedan depender del mecanismo de generación de la precipitación dominante. La aplicación de técnicas de análisis fractal se ha llevado a cabo a datos de lluvia registrados en el área metropolitana de Barcelona en el período 1994-2001, así como a una selección de eventos de lluvia sinópticos registrados en la misma ciudad en el período 1927-1992. Esta influencia se ha puesto de manifiesto también en el análisis de los efectos de la estacionalidad en el comportamiento multifractal de la lluvia en Barcelona (Rodríguez et al., 2013) y del comportamiento fractal en Cataluña (Meseguer-Ruiz et al., 2014). 
En otros ámbitos de la región mediterránea se han llevado a cabo trabajos en los que se ha determinado el valor de la dimensión fractal (Ghanmi et al., 2013). En este caso se calculó la dimensión fractal para diversas series temporales a distinta resolución (5-minutal y diaria) con diversas longitudes de las mismas (2,5 años las primeras, 137 años las segundas). Se identificaron tres estructuras auto-similares: microescala (de 5 minutos a 2 días) con una dimensión fractal de 1,44, mesoescala (de 2 días a una semana) y de escala sinóptica (de una semana a ocho meses) con dimensión fractal de 1,9. La interpretación de estos resultados sugiere que solo la microescala y la sinóptica son consistentes, mientras que la alta dimensión fractal relativa a la escala sinóptica podría verse afectada por la tendencia a la saturación. Kalauzi et al. (2009) proponen un estudio comparativo de la dimensión fractal no sólo de la precipitación, sino también de otras variables climatológicas, entre un ambiente mediterráneo, Veneto (Italia), y un ámbito completamente diferente, la provincia de Pastaza, en la Amazonía ecuatoriana. En este caso se han determinado los ritmos en los que se reproduce el principio de autosimilitud en cada serie, siendo mucho menor en la provincia de Pastaza (4,4 años), modulada por el ENSO, que en el ámbito mediterráneo de Veneto (10,3 años), donde se intuye la influencia del ciclo de actividad solar pero debe ser confirmada. Otra área donde se ha llevado a cabo un trabajo similar es la región de Tamil Nadu, en el extremo suroriental del subcontinente indio (Selvi et al., 2011). En este estudio, la determinación de la dimensión fractal se ha llevado a cabo a partir de datos entre 19022008 (no especificada la resolución temporal) con el método de Hurst, obteniendo un valor de $\mathrm{D}$ (dimensión fractal) de 1,7895, lo que implica que la precipitación en esta región es antipersistente.

\section{BASES DE DATOS Y METODOLOGÍA}

Se han utilizado las bases de datos de 48 observatorios de la red de estaciones automáticas de la Agencia Estatal de Meteorología (AEMet), de los cuales se han obtenido los datos de precipitación a resolución 10-minutal. Inicialmente, se dispuso de un total de 75 observatorios, pero se han descartado aquellas series que presentaban más de un $15 \%$ de missing values. Además, se han eliminado aquellos valores que aparecen como outliers, es decir, aquellos demasiado elevados para registrarse en 10 minutos, a fin de mantener en la mayor medida la homogeneidad de las series. Finalmente, el área de estudio queda cubierta razonablemente, tal y como se presenta en la Figura 1.

Por otra parte, se ha seleccionado un período común para los observatorios en los cuales se obtenía una buena homogeneidad, considerando el período entre 1997 y 2010.

El cálculo de la dimensión fractal (D) se lleva a cabo según el método box-counting, de la siguiente manera. A partir de registros pluviométricos a resolución 10-minutal, se considera el período de 10 minutos como el intervalo unitario de base para llevar a cabo el análisis. A continuación se han establecido períodos que contuvieran 1, 2, 3, 6, 12, 18, 24, 36, 48, 72, 144 y 288 intervalos unitarios, es decir, períodos de 10, 20 y 30 minutos, 1, 2, 3, 4, 6, 8, 12, 24 y 48 horas respectivamente, y se ha contabilizado en cuántos de ellos se registra alguna cantidad de precipitación. La utilización de intervalos de 10 minutos a 2 días responde a la intención de estudiar la persistencia o la ausencia de la misma en escalas temporales reducidas. Se define el valor de la dimensión fractal de la distribución temporal de la precipitación 
en base a la pendiente de la recta de regresión resultante de representar los pares de valores obtenidos a partir de los logaritmos naturales de $l$, extensión o longitud del intervalo en horas, y de $N$, número de intervalos con precipitación. En efecto, los logaritmos de esos pares de valores para cada observatorio se alinean con notable aproximación. La dimensión fractal $\mathrm{D}$ viene dada por $1+\mathrm{Q}$, donde $\mathrm{\square}$ es el valor absoluto de la pendiente de la recta de regresión. Los valores del coeficiente de determinación $\mathrm{r}^{2}$ de las rectas de regresión utilizadas para determinar D son muy elevados, tanto para el año completo como a nivel semestral, siendo el menor de ellos 0,9757 para el caso de Ibiza Aeropuerto en el semestre de verano.

Figura 1

LOCALIZACIÓN DE LOS OBSERVATORIOS UTILIZADOS

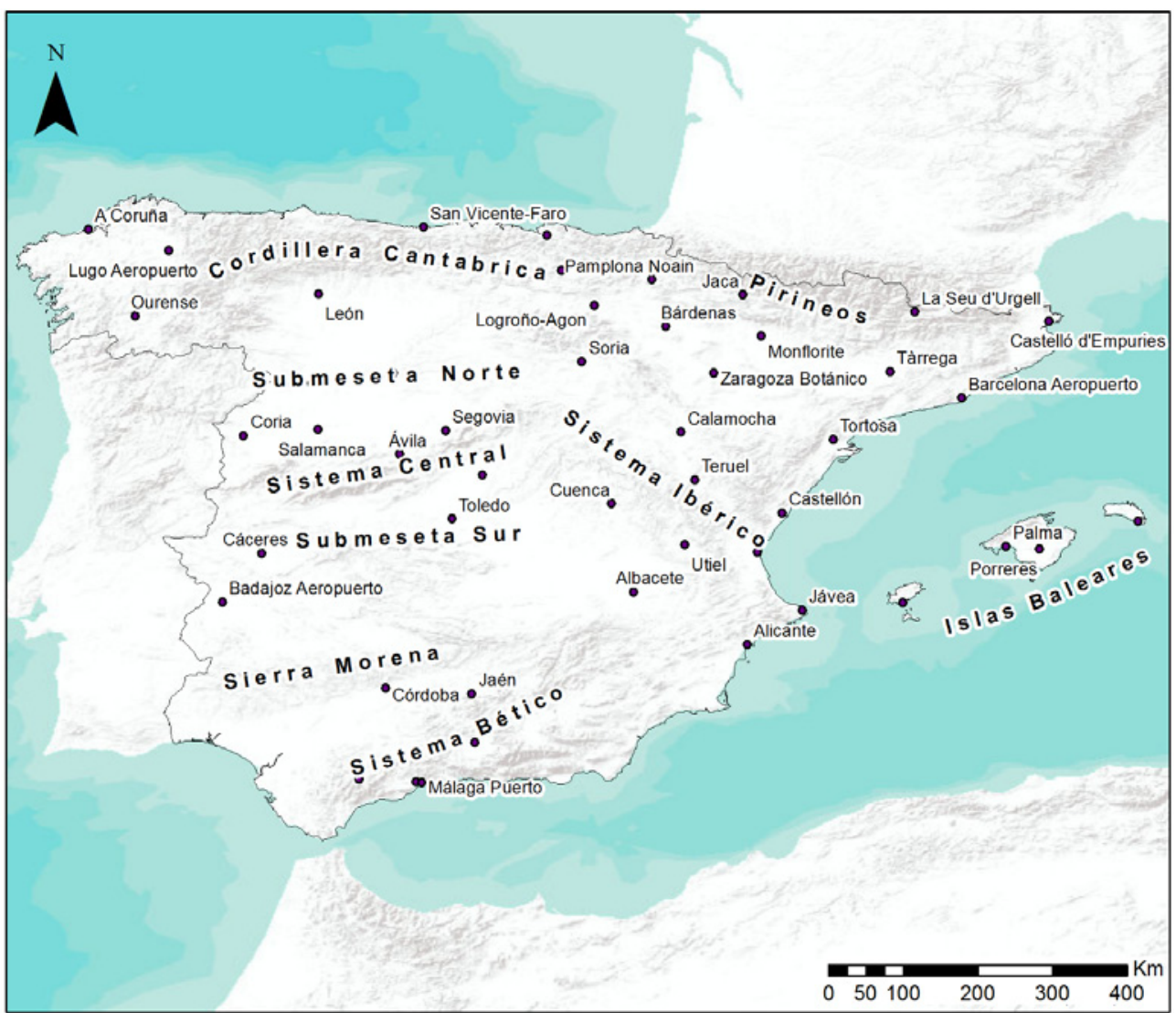

Fuente: elaboración propia.

Este proceso se ha llevado a cabo considerando la serie completa y los semestres cálido y frío, de tal manera que se han obtenido tres valores de D para cada observatorio. A modo de ilustración, para el caso del observatorio de Ávila queda tal y como se observa en la Tabla 1 y en la Figura 2. 
Tabla 1

VALORES OBTENIDOS DEL CONTEO DE INTERVALOS CON PRECIPITACIÓN PARA ÁVILA

\begin{tabular}{|c|c|c|c|}
\hline Ancho del intervalo & Año completo & Semestre cálido & Semestre frío \\
\hline 10 minutos & 17478 & 6358 & 11120 \\
\hline 20 minutos & 11443 & 4211 & 7232 \\
\hline 30 minutos & 9052 & 3368 & 5684 \\
\hline 1 hora & 6146 & 2362 & 3784 \\
\hline 2 horas & 4328 & 1703 & 2625 \\
\hline 3 horas & 3590 & 1418 & 2172 \\
\hline 4 horas & 3123 & 1250 & 1873 \\
\hline 6 horas & 2595 & 1060 & 1535 \\
\hline 8 horas & 2263 & 938 & 1325 \\
\hline 12 horas & 1892 & 791 & 1101 \\
\hline 24 horas & 1370 & 591 & 779 \\
\hline 48 horas & 993 & 433 & 560 \\
\hline
\end{tabular}

Así, para este caso, se obtienen unos valores de D de 1,5, 1,4655 y 1,5228 para el año completo, el semestre cálido y el semestre frío, respectivamente.

Figura 2

RECTAS DE REGRESIÓN PARA OBTENER LOS VALORES DE D EN ÁVILA

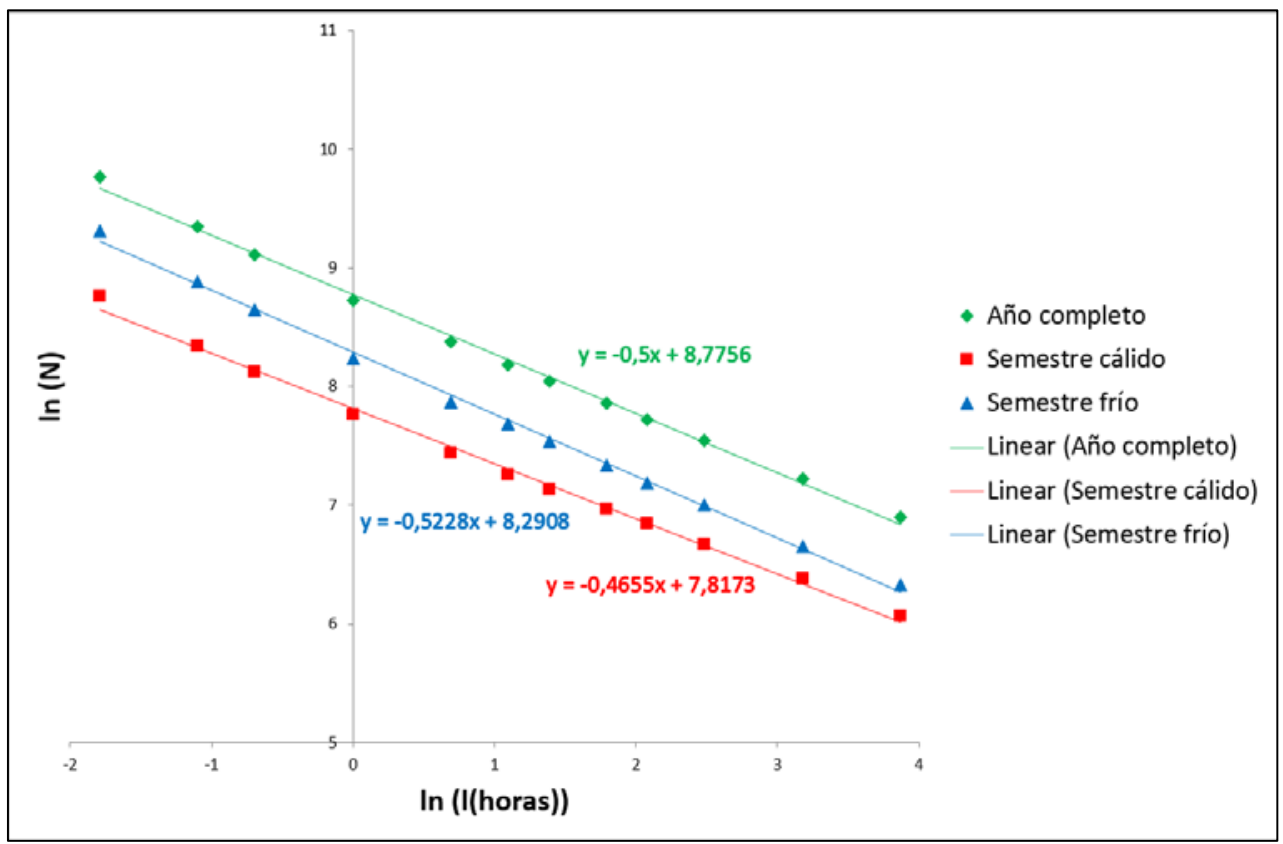

Fuente: elaboración propia. 
Así pues, a través del presente trabajo, se pretende dotar de un significado climático y geográfico al concepto de dimensión fractal aplicado a la distribución temporal de la precipitación, a partir de su comportamiento espacial en la España peninsular y Baleares.

\section{RESULTADOS}

Siguiendo la metodología propuesta anteriormente, se han obtenido los valores de D para todos los observatorios en el período anteriormente especificado para el año y los semestres cálido y frío (Tabla 2). Los valores de D para el año completo varían entre 1,4499 en Ibiza Aeropuerto y 1,6039 en Lugo Aeropuerto, mientras que para el semestre cálido los valores extremos se dan de nuevo en Ibiza Aeropuerto $(1,4253)$ y en Jaca $(1,5404)$. Para el semestre frío, el valor más bajo vuelve a registrarse en Ibiza Aeropuerto $(1,4600)$ y el más alto, en Lugo Aeropuerto $(1,6388)$. En la Tabla 3 se presentan los intervalos de confianza de las dimensiones fractales para cada caso, y es posible rechazar la hipótesis nula de que las series sean aleatorias, así como establecer que las diferencias observadas entre las dimensiones fractales de los semestres cálido y frío son significativas al $95 \%$.

Destaca el hecho de que siempre se obtienen valores más altos en el semestre frío que en el semestre cálido, aunque estas diferencias varían en magnitud en función del observatorio considerado. La diferencia más elevada se registra en Vitoria Aeropuerto, donde es de 0,1137 , mientras que la más baja es la que se da en Zaragoza Botánico, con un valor de 0,0045 .

El comportamiento espacial de los diferentes valores de D y de las diferencias obtenidas se presenta en las figuras 3 a 6 . En este sentido, a partir de los valores de D obtenidos para cada uno de los 48 observatorios utilizados, se ha realizado una interpolación para toda el área de estudio a través del procedimiento spline, que es un método mayoritariamente aceptado para este tipo de procesos en los que la variable no es estacionaria ni de primero ni de segundo orden, es decir, que tanto la media como la varianza varían de forma importante de unas regiones a otras del territorio analizado. Esto hace que el semivariograma experimental no sea adecuado como descriptor de la estructura de variabilidad espacial de la variable y, por tanto, el kriging, el método más utilizado para estos casos (Ninyerola et al., 2000; Vicente-Serrano et al., 2003; Ninyerola et al., 2007), no sea el más adecuado. En la Figura 3 se presenta la distribución espacial de la variable D del año completo, y se puede ver cómo los valores más elevados se concentran en dos zonas: en la vertiente cantábrica, con valores de D que se encuentran por encima de 1,56, y, en menor medida, en la parte meridional del valle del Guadalquivir. Los valores más bajos se encuentran en el archipiélago balear, en la región oriental peninsular y en el valle del Ebro, cuyos valores se sitúan entre 1,47 y 1,51, además de la parte occidental de la submeseta norte, donde aparece un área en la que se dan también valores bajos, cercanos al 1,50.

La distribución de los valores de D durante el semestre cálido (Figura 4) presenta algunas similitudes con la distribución de D para todo el año: los valores mínimos, entre 1,46 y 1,44 , se dan de nuevo en el archipiélago balear y en la franja del litoral mediterráneo, extendiéndose esta vez más hacia el sur y con menor presencia en el traspaís. Asimismo, los valores máximos, entre 1,50 y 1,52, se dan en la vertiente cantábrica, pero con unos valores muy inferiores a los que se daban para el caso de la precipitación anual. Los valo- 
res de D en el valle del Guadalquivir no son tan elevados, rondando 1,49. En la parte occidental de la submeseta norte siguen apareciendo valores bajos, alrededor de 1,46.

Los valores de D para el semestre frío (Figura 5) siguen una distribución que, en este caso, es muy similar a la del año completo. Efectivamente, los valores más altos, por encima de 1,60, se dan en la vertiente cantábrica y en Galicia, y los más bajos, inferiores a 1,52, aparecen en el oriente peninsular y en Baleares. La principal diferencia existente es que los valores que se dan en el valle del Guadalquivir no son tan elevados, situándose alrededor de 1,56 y 1,58 . Asimismo, los valores que se dan en la zona occidental de la submeseta norte no son tan bajos, ligeramente alejados de los mínimos peninsulares, entre 1,52 y 1,54.

Tabla 2

VALORES DE D PARA LOS OBSERVATORIOS ESTUDIADOS

\begin{tabular}{|c|c|c|c|c|}
\hline Observatorio & Año completo & Semestre cálido & Semestre frío & Diferencia \\
\hline A Coruña & 1,5629 & 1,5147 & 1,5919 & 0,0772 \\
\hline Albacete & 1,4941 & 1,4847 & 1,5005 & 0,0158 \\
\hline Alicante & 1,4710 & 1,4379 & 1,4895 & 0,0516 \\
\hline Ávila & 1,5000 & 1,4655 & 1,5228 & 0,0573 \\
\hline Badajoz Aeropuerto & 1,5183 & 1,4791 & 1,5352 & 0,0561 \\
\hline Barcelona Aeropuerto & 1,5071 & 1,4773 & 1,5307 & 0,0534 \\
\hline Bárdenas & 1,4933 & 1,4652 & 1,5118 & 0,0466 \\
\hline Bilbao Aeropuerto & 1,5827 & 1,5181 & 1,6276 & 0,1095 \\
\hline Cáceres & 1,5464 & 1,4863 & 1,5731 & 0,0868 \\
\hline Calamocha & 1,4805 & 1,4777 & 1,4843 & 0,0066 \\
\hline Castelló d'Empuries & 1,5161 & 1,4606 & 1,5576 & 0,0970 \\
\hline Castellón & 1,5075 & 1,4551 & 1,5475 & 0,0924 \\
\hline Córdoba Aeropuerto & 1,5605 & 1,4888 & 1,5864 & 0,0976 \\
\hline Coria & 1,5644 & 1,4953 & 1,5950 & 0,0997 \\
\hline Cuenca & 1,5468 & 1,5220 & 1,5622 & 0,0402 \\
\hline Granada & 1,5414 & 1,4709 & 1,5700 & 0,0991 \\
\hline Ibiza Aeropuerto & 1,4499 & 1,4253 & 1,4600 & 0,0347 \\
\hline Jaca & 1,5848 & 1,5404 & 1,6178 & 0,0774 \\
\hline Jaén & 1,5573 & 1,4793 & 1,5912 & 0,1119 \\
\hline Jávea & 1,5101 & 1,4880 & 1,5282 & 0,0402 \\
\hline La Seu d'Urgell & 1,5036 & 1,4834 & 1,5253 & 0,0419 \\
\hline León & 1,5578 & 1,4918 & 1,5916 & 0,0998 \\
\hline Logroño-Agon & 1,4961 & 1,4512 & 1,5287 & 0,0775 \\
\hline Lugo Aeropuerto & 1,6039 & 1,5443 & 1,6388 & 0,0945 \\
\hline Málaga Centro Meteo & 1,5595 & 1,4897 & 1,5844 & 0,0947 \\
\hline Málaga Puerto & 1,5376 & 1,4619 & 1,5647 & 0,1028 \\
\hline Menorca Aeropuerto & 1,4680 & 1,4323 & 1,4935 & 0,0612 \\
\hline Monflorite & 1,5223 & 1,4783 & 1,5559 & 0,0776 \\
\hline Ourense & 1,5704 & 1,5074 & 1,6038 & 0,0964 \\
\hline
\end{tabular}




\begin{tabular}{|c|c|c|c|c|}
\hline Palma & 1,4988 & 1,4568 & 1,5190 & 0,0622 \\
\hline Pamplona Noain & 1,5487 & 1,4858 & 1,5877 & 0,1019 \\
\hline Porreres & 1,4966 & 1,4550 & 1,5146 & 0,0596 \\
\hline Madrid Retiro & 1,5432 & 1,4868 & 1,5759 & 0,0891 \\
\hline Ronda Instituto & 1,5832 & 1,5088 & 1,6082 & 0,0994 \\
\hline Salamanca & 1,5075 & 1,4740 & 1,5288 & 0,0548 \\
\hline San Vicente-Faro & 1,5839 & 1,5267 & 1,6218 & 0,0951 \\
\hline Segovia & 1,5105 & 1,4741 & 1,5339 & 0,0598 \\
\hline Soria & 1,5190 & 1,4722 & 1,5545 & 0,0823 \\
\hline Tàrrega & 1,4732 & 1,4566 & 1,4935 & 0,0369 \\
\hline Teruel & 1,4856 & 1,4809 & 1,4906 & 0,0097 \\
\hline Toledo & 1,5047 & 1,4687 & 1,5258 & 0,0571 \\
\hline Tortosa & 1,5167 & 1,4782 & 1,5327 & 0,0545 \\
\hline Utiel & 1,5058 & 1,4688 & 1,5317 & 0,0629 \\
\hline Valencia & 1,5258 & 1,4886 & 1,5527 & 0,0641 \\
\hline Valladolid & 1,5261 & 1,4738 & 1,5579 & 0,0841 \\
\hline Vitoria Aeropuerto & 1,5559 & 1,4837 & 1,5974 & 0,1137 \\
\hline Zamora & 1,5020 & 1,4618 & 1,5266 & 0,0648 \\
\hline Zaragoza Botánico & 1,5154 & 1,5120 & 1,5165 & 0,0045 \\
\hline
\end{tabular}

Tabla 3.

VALORES DE LOS INTERVALOS DE CONFIANZA DE D AL 95\%

\begin{tabular}{|c|c|c|c|}
\hline Año completo & Semestre cálido & Semestre frío & Diferencia \\
\hline $1,4543-1,5986$ & $1,4315-1,5303$ & $1,4676-1,6340$ & $0,0132-0,1267$ \\
\hline
\end{tabular}

De la misma manera, conviene conocer cuál es la distribución espacial de las diferencias existentes entre los valores de D de ambos semestres (Figura 6), y es que ésta puede ser representativa de los diferentes mecanismos que se encuentren en el origen de la precipitación de un semestre a otro. El comportamiento espacial de las diferencias de D en ambos semestres no sigue unos patrones tan evidentes como en los casos anteriores. Se aprecian zonas en las que la diferencia del semestre frío menos el semestre cálido es elevada, próxima o superior a 0,1 , correspondiendo al valle del Guadalquivir y la mayor parte de la vertiente cantábrica. En otras zonas, estas diferencias aparecen atenuadas o son prácticamente inexistentes, del orden de 0,004. Se dan mayoritariamente en el este peninsular y en el interior de Cataluña. Parte del archipiélago balear y algunos puntos de Cataluña y de la Comunidad Valenciana son una excepción a este hecho, y es que en el extremo noreste de la primera y en el norte e interior de la segunda las diferencias aparecen más acentuadas, cercanas a $0,1, y$ las islas de Mallorca y Menorca en el archipiélago balear, con valores que rondan el 0,04. El sector occidental de la submeseta norte, en el que se suelen registrar valores bajos de D tanto a nivel anual como semestrales, presenta en este caso unas diferencias entre semestres que son también moderadas, pero que no tanto como en el sector oriental peninsular, alrededor de 0,03 y 0,04 . 
Figura 3

DISTRIBUCIÓN ESPACIAL DE LA DIMENSIÓN FRACTAL DE LA PRECIPITACIÓN ANUAL EN LA ESPAÑA PENINSULAR Y BALEARES

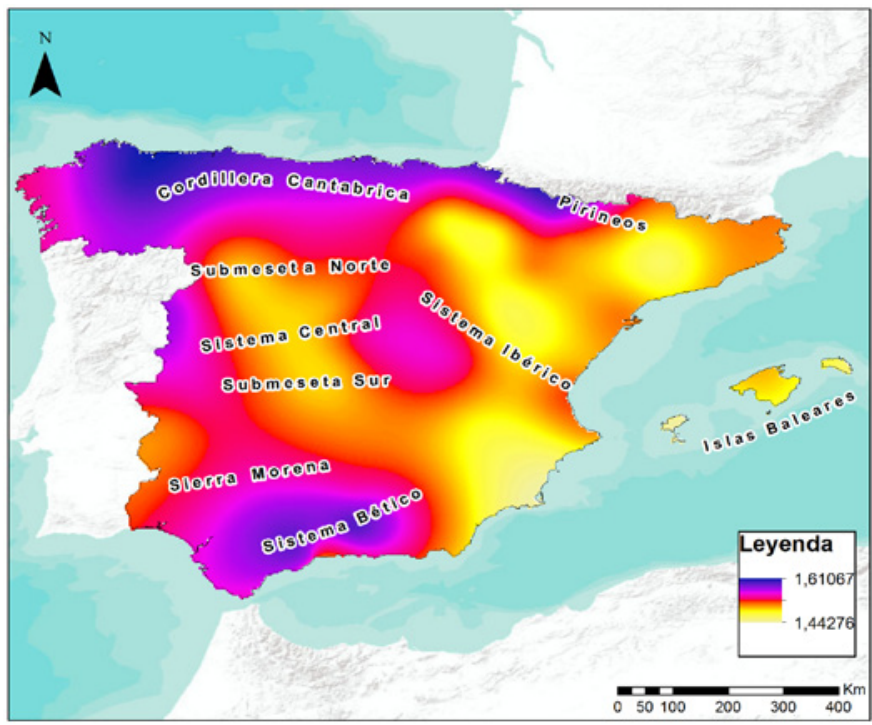

Fuente: elaboración propia.

Figura 4

DISTRIBUCIÓN ESPACIAL DE LA DIMENSIÓN FRACTAL DE LA PRECIPITACIÓN DEL SEMESTRE CÁLIDO EN LA ESPAÑA PENINSULAR Y BALEARES

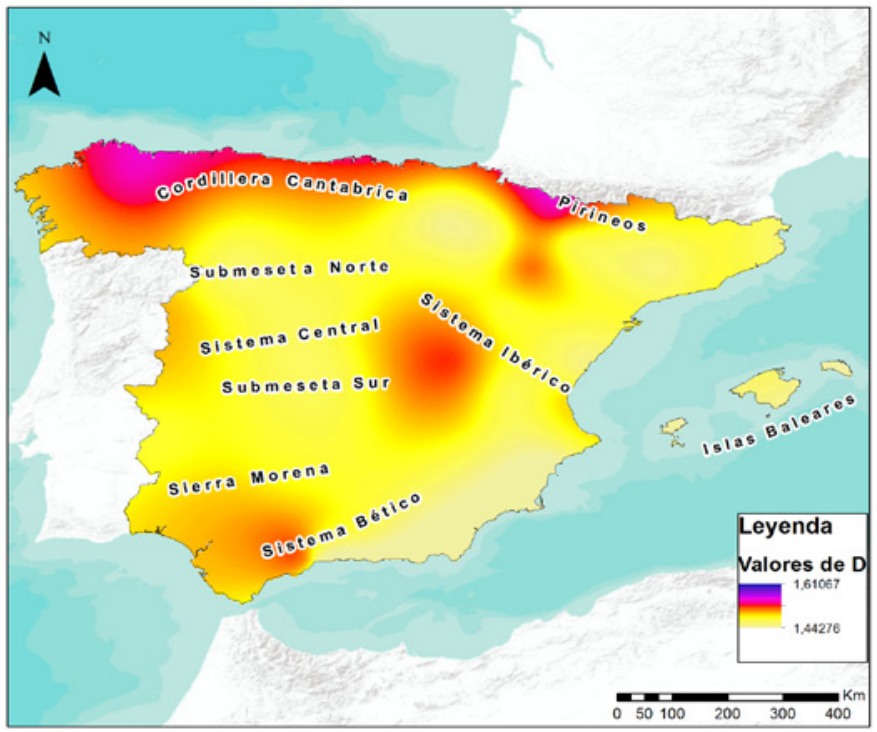

Fuente: elaboración propia. 
Figura 5

DISTRIBUCIÓN ESPACIAL DE LA DIMENSIÓN FRACTAL DE LA PRECIPITACIÓN DEL SEMESTRE FRIOO EN LA ESPAÑA PENINSULAR Y BALEARES

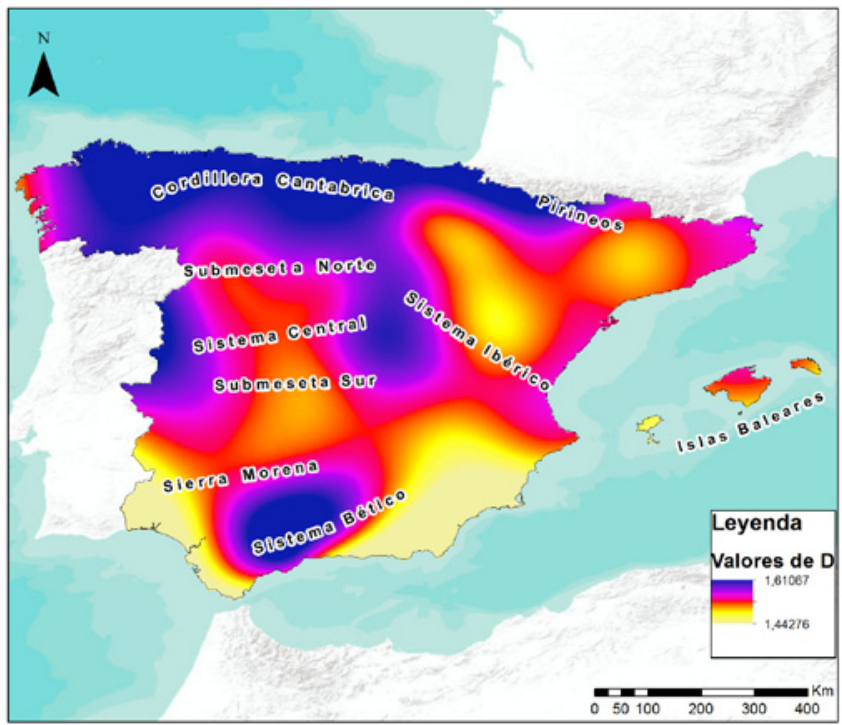

Fuente: elaboración propia.

Figura 6

DISTRIBUCIÓN ESPACIAL DE LA DIFERENCIA ENTRE LA DIMENSIÓN FRACTAL DE LA PRECIPITACIÓN DE LOS SEMESTRES FRÍO Y CÁLIDO EN LA ESPAÑA PENINSULAR Y BALEARES

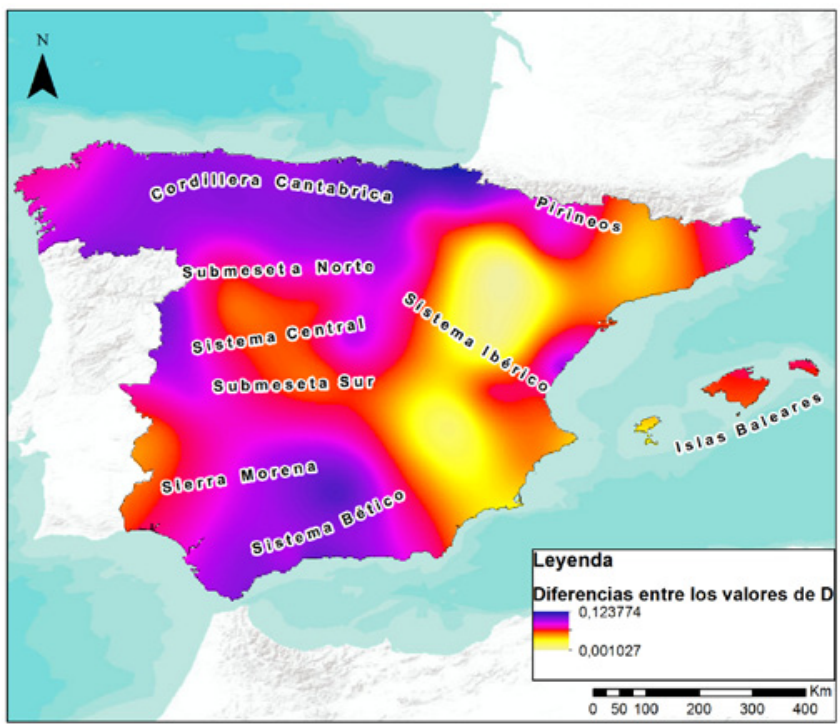

Fuente: elaboración propia. 


\section{DISCUSIÓN Y CONCLUSIONES}

Los valores de la dimensión fractal D que se han obtenido para los 48 observatorios estudiados oscilan entre 1,4499 y 1,6039 a nivel anual, entre 1,4253 y 1,5404 para el semestre cálido y 1,4600 y 1,6388 para el semestre frío. En todos los observatorios estudiados se da que el valor de D para el semestre cálido es inferior al obtenido para el año completo, y que el valor de D para el semestre frío es superior al del año completo.

Estos valores de D no son comparables con otros trabajos publicados sobre áreas de estudio similares (Meseguer-Ruiz et al., 2012; Meseguer-Ruiz et al., 2014), ya que las resoluciones temporales de las series utilizadas son diferentes. En el caso de los dos trabajos anteriores, la resolución es 30-minutal, y en el caso del presente trabajo, la resolución temporal es 10-minutal. Esto provoca que los datos de D obtenidos en los otros trabajos sean normalmente inferiores, ya que no existen valores de conteo para intervalos de 10 y de 20 minutos, con lo que, al obtener los correspondientes logaritmos naturales, y trazar la recta de regresión, el valor absoluto de la pendiente de la misma es inferior y, por lo tanto, también lo es el valor de D.

Breslin et al. (1999) proponen un método alternativo al box-counting y al análisis R/S de Hurst para el cálculo de la dimensión fractal de una serie temporal de precipitación basado en la variación de los totales mensuales de precipitación. Al margen de que las escalas temporales de ese estudio y del presente son diferentes, lo que se constata en ese estudio es la preferencia de esa metodología a la hora de calcular la dimensión fractal, y esto podría ser así únicamente en casos de datos mensuales, no a resolución 10-minutal, ya que muchos de los valores de la serie son nulos y además se encuentran muy seguidos, y por lo tanto, la variación en este tipo de series sería a menudo nula y, por lo tanto, no aplicable esta metodología.

Los valores de D obtenidos para Túnez en Ghanmi et al. (2013) son comparables a los que se han obtenido en el presente trabajo, ya que fueron calculados a partir de series de precipitación a resolución 5-minutal y siguiendo un método de box-counting. Estos valores se sitúan alrededor de 1,44 , lo que los hace muy similares a los que se obtienen en las áreas con menor precipitación de la España peninsular (Levante, Sureste ibérico y valle del Ebro) y Baleares (1,4499 en Ibiza).

Los valores de D siempre son inferiores en el semestre cálido respecto al semestre frío. Es sabido que la lluvia durante el segundo caso está más asociada a sistemas frontales, que dan lugar a una precipitación preferentemente estratiforme, más continuada en el tiempo, mientras que en el primer caso la precipitación tiene un carácter más convectivo y concentrado. Así pues, unos valores de $\mathrm{D}$ bajos, inferiores a 1,5, parecen ir asociados a precipitaciones de carácter convectivo, mientras que valores de $\mathrm{D}$ elevados, se vincularían a una lluvia más continua y menos concentrada. Esto es así en un gran número de observatorios. El valor de D durante el semestre cálido sigue siendo elevado en el norte, y estos valores podrían estar asociados tanto a precipitaciones de origen frontal, como de origen convectivo.

No se conocen trabajos que hayan presentado el comportamiento espacial de la dimensión fractal de series temporales de precipitación más allá del estudio de Meseguer-Ruiz (2014), por lo que establecer una comparación sobre este indicador es complicado. Sí que se puede establecer, basándose en el conocimiento del comportamiento de la pluviometría en la España peninsular y Baleares, algunos patrones sobre las variaciones de D en el espacio y en función del período estudiado. 
En base a los resultados obtenidos, se puede afirmar que, a nivel anual, las dimensiones fractales más elevadas se sitúan en el norte peninsular y en el valle del Guadalquivir, áreas en las que se recogen importantes cantidades de precipitación en invierno y a lo largo de todo el año en la primera región. Los menores valores de D se dan en el litoral mediterráneo oriental y en el valle del Ebro, donde las precipitaciones suelen ser más escasas y de marcado carácter estacional.

Durante el semestre cálido, es posible ver cómo en la región norte se mantienen valores de $\mathrm{D}$ elevados respecto a los que se dan en el resto del área de estudio, mientras que en el valle del Guadalquivir estos valores son inferiores. En esta época del año, al norte de la Península Ibérica continúan llegando, aunque con menor frecuencia, frentes polares provenientes del oeste, mientras que en el sur peninsular, el anticiclón de las Azores, habiéndose desplazado hacia el norte respecto a su posición habitual, juega un papel de bloqueador de las borrascas atlánticas, con lo que disminuyen las precipitaciones hasta hacerlas casi inexistentes en el centro del verano. El litoral mediterráneo oriental sigue presentando valores bajos, ya que se trata de una región donde las precipitaciones en verano son en general escasas. En el sector pirenaico occidental aparecen valores relativamente elevados, ya que se registran importantes precipitaciones en los meses estivales. En el centro de la Península, los valores de D son bajos, pero no tanto como en el área mediterránea.

Durante el semestre frío, se aprecia cómo la región norte sigue presentando los valores de D más elevados, y que la región mediterránea continúa mostrando los más bajos. En el valle del Guadalquivir se da el segundo máximo del área de estudio, con precipitaciones relativamente abundantes. Los principales tipos de tiempo asociados a precipitación en la Península Ibérica y Baleares durante el semestre frío son los que comportan el paso de frentes fríos vinculados a borrascas atlánticas. Estos frentes suelen afectar a la Península por el oeste, dejando importantes precipitaciones a su paso, degradándose a medida que avanzan hacia el este. Normalmente, apenas dejan precipitación cuando llegan al área mediterránea. En el valle del Guadalquivir, las precipitaciones de invierno se asocian a borrascas estancadas en el golfo de Cádiz, que dejan grandes cantidades de agua. Sin embargo, el invierno presenta, en muchos sectores de la vertiente mediterránea oriental, el mínimo pluviométrico secundario, lo que explicaría los valores bajos de $\mathrm{D}$, con lluvia poco persistente en el tiempo.

Las diferencias existentes entre los semestres frío y cálido son máximas en las áreas en las que los valores de D son más altos, independientemente de la época del año considerada, y son mínimas en las regiones con una dimensión fractal más baja. Esto implica que existen mayores diferencias entre ambos períodos en la frecuencia y persistencia de la lluvia en las regiones con valores de $\mathrm{D}$ elevados que en las regiones con valores de D bajos. Esto refleja el hecho de que durante el semestre cálido la llegada de sistemas frontales a la Península Ibérica es mucho menos frecuente que en los meses del semestre frío. Es interesante señalar que las menores diferencias de D entre los semestres frío y cálido se producen en los observatorios de Zaragoza Botánico, Calamocha y Teruel, un triángulo donde la precipitación en el trimestre estival es igual o superior a la del invernal.

La desigual distribución espacial de los observatorios utilizados puede provocar que los resultados obtenidos aparezcan sesgados. No disponer de ninguna estación de la zona más seca del área de estudio (Murcia y Almería), así como tampoco del suroeste peninsular (Huelva y Cádiz) puede haber afectado los resultados obtenidos. Asimismo, si se hubiera dispuesto de observatorios de la fachada septentrional de las islas del archipiélago balear, 
hubiera sido interesante contrastar si los valores de D en estos casos son superiores a los de Ibiza, Palma, Porreres o Menorca. De esta manera, hubiera quedado en evidencia el efecto orográfico y el sesgo que implica la irregular distribución de puntos de medida.

En definitiva, se puede concluir que los valores de la dimensión fractal en series temporales en la Península Ibérica y Baleares dependen de la localización y de la pluviometría del observatorio. A nivel anual, el valor más alto se halló en el observatorio de Lugo Aeropuerto $(1,6039)$, y el valor más bajo en Ibiza Aeropuerto (1,4499). En el semestre cálido, los valores que se obtuvieron fueron siempre inferiores a aquellos del año completo, variando entre 1,5405 en Jaca y 1,4253 de nuevo en Ibiza Aeropuerto. Por su parte, durante el semestre frío, los valores siempre fueron superiores a los obtenidos para el año completo, con índices que van desde 1,4600 en Ibiza Aeropuerto hasta 1,6388 en Lugo Aeropuerto. A menores valores de $\mathrm{D}$, en mayor medida se cumple la característica de autosimilitud en la distribución temporal de la precipitación, e, inversamente, a mayor valor de $\mathrm{D}$, menos se cumple, coincidiendo con los resultados presentados en Selvi et al. (2011).

A partir de la distribución espacial de la dimensión fractal se han podido identificar cuatro regiones dentro del área de estudio: una región norte, donde se dan los valores más elevados de D, tanto a nivel anual como en los diferentes semestres; una región sur, con valores también elevados en el año completo, así como durante el semestre frío, pero que durante el semestre cálido presenta valores más bajos; una región central, correspondiente a la Meseta, con valores siempre relativamente bajos, tanto para el año completo como para ambos semestres, pero con diferencias marcadas entre ellos (del orden de 0,06); y una región mediterránea y del valle del Ebro, donde se hallan los valores más bajos de D, independientemente de la época del año considerada.

\section{AGRADECIMIENTOS}

La presente investigación se enmarca en el seno de los proyectos FONDECYT 11160059 de la Comisión Nacional de Ciencia y Tecnología del Gobierno de Chile, y WEMOTOR (CSO2014-55799-C2-1-R) del Ministerio de Economía y Competitividad del Gobierno de España, así como en el pro $\neg$ grama del Grupo de Climatología (2014SGR300, Generalitat de Catalunya) de la Universidad de Barcelona. Se agradece especialmente al Convenio de Desempeño UTA-MINEDUC del Gobierno de Chile, así como a la Agencia Estatal de Meteorología de España por la cesión de los datos meteo $\neg$ rológicos, sin los cuales este trabajo no habría podido llevarse a cabo.

\section{REFERENCIAS BIBLIOGRÁFICAS}

AMARO, I.R., DEMEY, J.R. y MACCHIAVELLI, R. (2004): «Aplicación del análisis R/S de Hurst para estudiar las propiedades fractales de la precipitación en Venezuela». Interciencia, $\mathrm{n}^{\circ}$ 29-11, 617-620.

BODRI, L. (1994): «Fractal Analysis of Climatic Data: Mean Annual Temperature Records in Hungary». Theoretical and Applied Climatology, $\mathrm{n}^{\circ}$ 49, 53-57.

BRESLIN, M.C. y BELWARD, J.A. (1999): "Fractal dimensions for rainfall time series». Mathematics and Computers in Simulation, $\mathrm{n}^{\circ} 48,437-446$. 
CASANUEVA, A., RODRÍGUEZ-PUEBLA, C., FRÍAS, M.D. y GONZÁLEZ-REVIRIEGO, N. (2014): «Variability of extreme precipitation over Europe and its relationships with teleconnection patterns», Hydrology and Earth System Sciences, n 18, 709-725.

DE LUIS, M., BRUNETTI, M., GONZALEZ-HIDALGO, J.C., LONGARES, L.A. y MARTIN-VIDE, J. (2010): «Changes in seasonal precipitation in the Iberian Peninsula during 1946-2005», Global and Planetary Change, $\mathrm{n}^{\circ}$ 74, 27-33.

DUNKERLEY, D. (2008): «Rain event properties in nature and in rainfall simulation experiments: a comparative review with recommendations for increasingly systematic study and reporting». Hydrological Processes, $\mathrm{n}^{\circ} 22$, 4415-4435.

DUNKERLEY, D.L. (2010): «How do the rain rates of sub-events intervals such as the maximum 5- and 15-min rates $\left(\mathrm{I}_{5}\right.$ or $\left.\mathrm{I}_{30}\right)$ relate to the properties of the enclosing rainfall event? ». Hydrological Processes, $\mathrm{n}^{\circ} 24,2425-2439$.

GAO, J. y XIA, Z. (1996): «Fractals in physical geography». Progress in Physical Geography, $\mathrm{n}^{\circ} 20$ (2), 178-191.

GAO, M. y HOU, X. (2012): «Trends and Multifractals Analyses of Precipitation Data from Shandong Peninsula, China». American Journal of Environmental Sciences, $\mathrm{n}^{\circ} 8$ (3), 271-279.

GARCÍA MARÍN, A.P. (2007): Análisis multifractal de series de datos pluviométricos en Andalucía. Tesis doctoral. Departamento de Ingeniería Rural, Escuela Técnica Superior de Ingenieros Agrónomos y Montes. Universidad de Córdoba.

GARCÍA-MARÍN, A.P., JIMÉNEZ-HORNERO, F.J. y AYUSO-MUÑOZ, J.L. (2008): «Universal multifractal description of an hourly rainfall time series from a location in southern Spain». Atmósfera, $\mathrm{n}^{\circ} 21$ (4), 347-355.

GHANMI, H., BARGAOUI, Z. y MALLET, C. (2013): «Investigation of the fractal dimension of rainfall occurrence in a semi-arid Mediterranean climate». Hydrological Sciences Journal, $\mathrm{n}^{\circ} 58$ (3), 483-497.

GNEITING, T. y SCHALTER, M. (2004): «Stochastic models that separate fractal dimension and the Hurst effect», Society for Industrial and Applied Mathematics Review, $\mathrm{n}^{\circ} 46$, 269-282.

GONZALEZ-HIDALGO, J.C., LOPEZ-BUSTINS, J.A., ŠTEPÁNEK, P., MARTIN-VIDE, J. y DE LUIS, M. (2009): «Monthly precipitation trends on the Mediterranean fringe of the Iberian Peninsula during the second half of the twentieth century (1951-2000)», International Journal of Climatology, $\mathrm{n}^{\circ} 29,1415-1429$.

GOODCHILD, M.F. (1980): «Fractals and the accuracy of geographical measures». Mathematical Geology, $\mathrm{n}^{\circ} 12$ (2), 85-98.

GOODCHILD, M.F. y MARK D.M. (1987): «The Fractal Nature of Geographic Phenomena». Annals of the Association of American Geographers, $\mathrm{n}^{\circ} 77$ (2), 265-278.

GOODESS, C.M. y JONES, P.D. (2002): «Links between circulation and changes in the characteristics of Iberian rainfall», International Journal of Climatology, n 22, 1593-1615.

GUSEV, A.A., PONOMOREVA, V.V., BRAITSEVA, O.A., MELEKESTSEV, I.V. y SULERZHITSKY, L.D. (2003): «Great explosive eruptions on Kamchatka during the last 10000 years: Self-similar irregularity of the output of volcanic products». Journal of Geophysical Research-Solid Earth, ${ }^{\circ} 108$, art. No. 2126

HASTINGS, H.M. y SUGIHARA, G. (1994): Fractals: A User's Guide for the Natural Sciences. Oxford. Ed. Oxford University Press. 
KALAUZI, A., CUKIC, M., MILLÁ, H., BONAFONI, S. y BIONDI, R. (2009): «Comparison of fractal dimension oscillations and trends of rainfall data from Pastaza Province, Ecuador and Veneto, Italy». Atmospheric Research, $\mathrm{n}^{\circ}$ 93, 673-679.

KHAN, M.S. y SIDDIQUI, T.A. (2012): «Estimation of fractal dimension of a noisy time series». International Journal of Computer Applications, $\mathrm{n}^{\circ} 45$ (10), 1-6.

KING, "M.R. (2005): «Fractal analysis of eight glacial cycles from an Antarctic ice core». Chaos, Solitons and Fractals, $\mathrm{n}^{\circ} 25,5-10$.

KUTIEL, H. y TRIGO, R.M. (2014): «The rainfall regime in Lisbon in the last 150 years». Theoretical and Applied Climatology, $\mathrm{n}^{\circ} 118$ (3), 387-403.

LANGOUSIS, A., VENEZIANO, D., FURCOLO, P. y LEPORE, C. (2009): «Multifractal rainfall extremes: Theoretical analysis and practical estimation». Chaos, Solitons and Fractals, n 39, 1182-1194.

LÓPEZ LAMBRAÑO, A.A. (2012): Análisis multifractal y modelación de la precipitación, Tesis doctoral. Facultad de Ingeniería. Universidad Autónoma de Querétaro.

MANDELBROT, B.B. (1967): «How long is the coast of Britain? Statistical self-similarity and fractional dimension». Science, $\mathrm{n}^{\circ} 156,636-638$.

MANDELBROT, B.B. (1976): The fractal Geometry of Nature. New York. W.H. Freeman and Company.

MARTÍN-VIDE, J. y ESTRADA MATEU, J. (1998): «Una nueva propuesta metodológica de regímenes pluviométricos estacionales para la Península Ibérica». Nimbus, nº 1-2, 85-92.

MARTIN-VIDE, J. y LOPEZ-BUSTINS, J.A. (2006): «The Western Mediterranean Oscillation and rainfall in the Iberian Peninsula», International Journal of Climatology, n⿳2 26, 1455-1475.

MARTÍN-VIDE, J. (2008): «La nueva realidad del calentamiento global. Un decálogo del cambio climático». Scripta Nova, Vol. XII, 270 (23).

MAZZARELLA, A. y RAPETTI, F. (2004): «Scale-invariance laws in the recurrence interval of extreme floods: an application to the upper Po river valley (northern Italy)». Journal of Hydrology, $\mathrm{n}^{\circ}$ 288, 264-271.

MESEGUER-RUIZ, O. y MARTÍN-VIDE, J. (2012): «Análisis de la fractalidad temporal de la precipitación en Cataluña durante 2010 y 2011» en Cambio climático. Extremos e impactos (Rodríguez Puebla, C., Ceballos Barbancho, A., González Reviriego, N., Morán Tejeda, E. y Hernández Encinas A., Coords.). Salamanca, Publicaciones de la Asociación Española de Climatología, 539-547.

MESEGUER-RUIZ, O. y MARTÍN-VIDE, J. (2014): «Análisis de la fractalidad temporal de la precipitación en Cataluña, España (2010) ». Investigaciones Geográficas, n 47, 41-52.

MORENO, M.C. y MARTÍN-VIDE, J. (1986): «Estudio preliminar sobre las tendencias de la precipitación anual en el sur de la Península Ibérica: el caso de Gibraltar». II Simposio sobre el Agua en Andalucía, Departamento de Hidrología, Universidad de Granada, 37-44.

NINYEROLA, M., PONS, X. y ROURE, J.M. (2000): «A methodological approach of climatological modelling of air temperature and precipitation through GIS techniques». International Journal of Climatology, $\mathrm{n}^{\circ} 20,1823-1841$.

NINYEROLA, M., PONS, X. y ROURE, J.M. (2007): «Monthly precipitation mapping of the Iberian Peninsula using spatial interpolation tools implemented in a Geographic Information System». Theoretical and Applied Climatology, ${ }^{\circ} 89,195-209$. 
NUNES, S.A., ROMANI, L.A.S., AVILA, A.M.H., COLTRI, P.P., TRAINA, C., CORDEIRO, R.L.F., DE SOUSA, E.P.M. y TRAINA, A.J.M. (2011): «Fractal-based Analysis to Identify Trend Changes in Multiple Climate Time Series». Journal of Information and Data Management, $\mathrm{n}^{\circ}$ 2, 51-57.

NUNES, S.A., ROMANI, L.A.S., AVILA, A.M.H., COLTRI, P.P., TRAINA, C., CORDEIRO, R.L.F., DE SOUSA, E.P.M. y TRAINA, A.J.M. (2013): «Analysis of Large Scale Climate Data: How Well Climate Change Models and Data from Real Sensor Networks Agree?»; en Proceedings of the IW3C2 WWW 2013 Conference (Schwabe, D., Almeida, V., Glaser, H., Baeza-Yates, R. y Moon, S., cords.). IW3C2 2013, Rio de Janeiro, 517-526.

OÑATE RUBALCABA, J.J. (1997): «Fractal Analysis of Climatic Data: Annual Precipitation Records in Spain». Theoretical and Applied Climatology, $\mathrm{n}^{\circ}$ 56, 83-87.

PEITGEN, H.O., JÜRGENS, H. y SAUPE, D. (1992): Chaos and Fractals: New Frontiers of Science. New York, Springer.

PELLETIER, J.D. (1997): «Analysis and modelling of the natural variability of climate». Journal of Climate, $\mathrm{n}^{\circ} 10,1331-1342$.

PÉREZ, S.P., SIERRA, E.M., MASSOBRIO, M.J. y MOMO, F.R. (2009): «Análisis fractal de la precipitación anual en el este de la Provincia de la Pampa, Argentina». Revista de Climatología, $\mathrm{n}^{\circ}$ 9, 25-31.

RAIDL, A. (1996): «Estimating the fractal dimension, K-2-entropy, and the predictability of the atmosphere». Czechoslovak Journal of Physics, $\mathrm{n}^{\circ} 46,296-328$.

RANGARAJAN, G. y SANT, D.A. (1997): «A climate predictability index and its applications». Geophysical Research Letters, $\mathrm{n}^{\circ} 24,1239-1242$.

RANGARAJAN, G. y SANT, D.A. (2004): «Fractal dimensional analysis of Indian climatic dynamics». Chaos, Solitons and Fractals, ${ }^{\circ}$ 19, 285-291.

REHMAN, S. (2009): «Study of Saudi Arabian climatic conditions using Hurst exponent and climatic predictability index». Chaos, Solitons and Fractals, ${ }^{\circ} 39,499-509$.

REHMAN, S. y SIDDIQI, A.H. (2009): «Wavelet based Hurst exponent and fractal dimensional analysis of Saudi climatic dynamics». Chaos, Solitons and Fractals, $\mathrm{n}^{\circ} 40$, 1081-1090.

REISER, H. y KUTIEL, H. (2010): «Rainfall uncertainty in the Mediterranean: Intraseasonal rainfall distribution». Theoretical and Applied Climatology, $\mathrm{n}^{\circ}$ 100, 105-121.

RODRÍGUEZ, R., CASAS, M.C. y REDAÑO, A. (2013): «Multifractal analysis of the rainfall time distribution on the metropolitan area of Barcelona (Spain) ». Meteorology and Atmospheric Physics, $\mathrm{n}^{\circ} 121,181-187$.

RODRÍGUEZ-PUEBLA, C., ENCINAS, A.H., NIETO, S., y GARMENDIA, J. (1998): «Spatial and temporal patterns of annual precipitation variability over the Iberian Peninsula», International Journal of Climatology, nº 18, 299-316.

RODRÍGUEZ-PUEBLA, C. y NIETO, S. (2010): «Trends of precipitation over the Iberian Peninsula and the North Atlantic Oscillation under climate change conditions», International Journal of Climatology, $\mathrm{n}^{\mathrm{o}}$ 30, 1807-1815.

SÁENZ, J., ZUBILLAGA, J. y RODRÍGUEZ-PUEBLA, C. (2001): «Interannual variability of winter precipitation in northern Iberian Peninsula», International Journal of Climato$\log y, \mathrm{n}^{\mathrm{o}} 21,1503-1513$. 
SAHAY, J.D. y SREENIVASAN, K.R. (1996): «The search for a low-dimensional characterization of a local climate system». Philosophical Transactions of the Royal Society, $\mathrm{n}^{\circ}$ $354,1715-1750$.

SELVI, T. y SELVARAJ, S. (2011): «Fractal dimension analysis of Northeast monsoon of Tamil Nadu». Universal Journal of Environmental Research and Technology, $\mathrm{n}^{\circ} 1$ (2), 219-221.

SCHROEDER, K., GARCÍA-LAFUENTE, J., JOSEY, S.A., ARTALE, V., BUONGIORNO NARDELLI, B., CARRILLO, A., GAČIĆ, M., GASPARINI, G.P., HERRMAN, M., LIONELlO, P., LUDWIG, W., MILLOT, C., ÖZSOKY, E., PISACANE, G., SÁNCHEZ-GARRIDO, J.C., SANNINO, G., SANTOLERI, R., SOMOT, S., STRUGLIA, M., STANEV, E., TAUPIER-LETAGE, I., TSIMPLIS, M.N., VARGAS-YÁÑEZ, M., ZERVAKIS, V. y ZODIATIS, G. (2012): «Circulation of the Mediterranean Sea and it's Variability» en The Climate of the Mediterranean Region. From the Past to the Future (Pionello, P., Coord.). London, Ed. Elsevier Insights, 187-256.

SIVAKUMAR, B. (2001): «Is a chaotic multi-fractal approach for rainfall possible?». Hydrological Processes, $\mathrm{n}^{\circ} 15,943-955$.

VALDEZ-CEPEDA, R.D., HERNANDEZ-RAMIREZ, D., MENDOZA, B., VALDESGALICIA, J. y MARAVILLA, D. (2003): «Fractality of monthly extreme minimum temperature». Fractals, ${ }^{\circ} 11,137-144$.

VENEZIANO, D. y FURCOLO, P. (2002): «Multifractality of rainfall and scaling of intensity-duration-frequency curves». Water Resources Research, $\mathrm{n}^{\circ} 38$ (12), 1306, doi: 10.1029/2001WR000372

VENEZIANO, D., LANGOUSIS, A. y FURCOLO, P. (2006): «Multifractality and rainfall extremes: A review». Water Resources Research, $\mathrm{n}^{\circ}$ 42, W06D15, doi: 10.1029/2005WR004716

VICENTE-SERRANO, S. M., SAZ-SÁNCHEZ, M.A. y CUADRAT, J.M. (2003): «Comparative analysis of interpolation methods in the middle Ebro Valley (Spain): application to annual precipitation and temperature». Climate Research, n $^{\circ} 24,161-180$.

WHEELER, D. y MARTÍN-VIDE, J. (1992): «Rainfall Characteristics of Mainland Europe's most Southerly Stations». International Journal of Climatology, nº12, 96-76.

ZHOU, X. (2004): Fractal and Multifractal Analysis of Runoff Time Series and Stream Networks in Agricultural Watersheds, Tesis doctoral, Virginia Polytechnic Institute and State University. 